Research Article

\title{
A Comprehensive Optimization Control Method for Hybrid Excitation Synchronous Motor
}

\author{
Mingming Huang, Quanzhen Huang ${ }^{D}$, Yang Zhang, and Xinjun Guo \\ School of Electrical Information Engineering, Henan University of Engineering, Zhengzhou, Henan 451191, China \\ Correspondence should be addressed to Quanzhen Huang; huang2004_susu@126.com
}

Received 24 February 2020; Accepted 11 June 2020; Published 3 July 2020

Academic Editor: Rafael Morales

Copyright (c) 2020 Mingming Huang et al. This is an open access article distributed under the Creative Commons Attribution License, which permits unrestricted use, distribution, and reproduction in any medium, provided the original work is properly cited.

\begin{abstract}
Hybrid excitation synchronous motor (HESM) offers the advantages of a convenient flux regulation and a wide range of available speeds. As such, it may be conveniently employed in the fields of electric vehicle and aerospace. In this paper, based on a suitable control strategy for vector and speed partition, we put forward a speed control method for HESM which shows optimal efficiency. On the one hand, our method aims at minimizing the copper loss by an optimal configuration of the $q$-axis current, the $d$-axis current, and the excitation current under the constraint voltage at the armature end. On the other hand, we conduct a comprehensive analysis of the effects of the flux-weakening speed coefficient on the operating performance of the motors and suggest a self-adaptive control method to regulate that coefficient and further improve the overall performance of the motors, including their speed-range regulation, their efficiency, and their dynamical properties. The proposed flux-weakening control method has been assessed by simulations and HESM-driven experiments. Results have confirmed the feasibility and optimality of our method.
\end{abstract}

\section{Introduction}

Hybrid excitation synchronous motor (HESM) represents a novel class of motors based on the permanent magnet synchronous motor (PMSM) and is characterized by a wider range of available speeds [1-3]. There are two magnetic potential sources in this kind of motor, with the permanent magnet providing a nearly constant magnetic field in the air gap and the excitation winding providing a magnetic field with variable magnitude and direction. The HESM is thus endowed with a large torque with small speed and may access a wide range of speeds. Therefore, HESM has extensive applications in the areas of electric vehicle, machine tools, and aerospace $[4,5]$.

The electromagnetic torque generated by the HESM is a nonlinear function of the $d$-axis current, the $q$-axis current, and the excitation current $[6,7]$. Therefore, a given electromagnetic torque may be generated by different current partitions, each one leading to a different total copper loss [8]. In order to enhance the performance of the motor, the control method of maximum torque per ampere (MTPA) used for PMSM is often adopted [9-11] to realize the optimal configuration of the armature and excitation currents. However, there is one more controllable excitation current in the HESM than in the PMSM, and thus the MTPA control method only provides copper loss minimization control for HESM [12], leaving room for further improvement.

For the hidden pole or the approximate hidden pole of the HESM, the authors of $[8,13]$ have proposed a vector control method based on the $d$-axis current $i_{d}=0$ combined with the copper loss minimization control strategy to optimize the motor efficiency. The disadvantage of the method is that, during the flux-weakening regulation, when the motor is operating at high speed, the flux-weakening effect on the $d$-axis current is not fully exploited, resulting in a limitation of the available motor speed range. The authors proposed a piecewise optimization control algorithm [14], which combines fuzzy control with particle swarm optimization to enhance the motor efficiency and stability. The shortcoming here is that, during the flux-weakening, the base-speed coefficient is constant and cannot maintain the optimal working point when the motor operating state 
changes. The flux-weakening method may be also applied while keeping constant the back electromotive force (back EMF) [13], and this makes the motor able to show a satisfactory operating performance, though the stability problem of [12] is not solved.

In this paper, an innovative efficiency optimization control method is proposed, which is combined with the vector control and speed partition control principles $[14,15]$. Our method aims at minimizing the copper loss by the motor, and it consists in exploiting two different control methods depending on the speed of the HESM. Upon splitting the possible values of the speed into two intervals, we employ the copper loss minimization control method with $i_{d}=0$ for "low speed," whereas for "high speed," the flux-weakening base-speed coefficient is adjusted by the selfadaptive method to realize the optimal configuration of the excitation and armature currents, as well as to achieve the efficiency optimization control. We also present a set of simulations and motor-driven experiments to show the validity and the efficiency of the proposed control method.

\section{Mathematical Model of HESM}

In a generic HESM, the $d$-q-axis coordinate is established by adopting the directional control method of the rotor magnetic field, whose air gap magnetic field is generated by the permanent magnetic body, $d$-axis current of the armature coil, and the current of the excitation coil. The effects of temperature fluctuations, iron loss, stray loss, magnetic field saturation, and other influences inside the motor are neglected. The HESM excitation current is assumed to have the same phase of the air gap magnetic flux generated by the permanent magnetic body. With these assumptions, the equivalent circuit model of the motor at the steady state is shown in Figure 1.

Based on the equivalent circuit model shown in Figure 1, the following basic equations of the HESM can be derived [14].

The circuit equations are

$$
\left[\begin{array}{l}
u_{d} \\
u_{q} \\
u_{f}
\end{array}\right]=\left[\begin{array}{ccc}
R_{s}+s L_{d} & -p \omega_{r} L_{q} & s M_{s f} \\
p \omega_{r} L_{d} & R_{s}+s L_{q} & p \omega_{r} M_{s f} \\
s M_{s f} & 0 & R_{f}+s L_{f}
\end{array}\right]\left[\begin{array}{c}
i_{d} \\
i_{q} \\
i_{f}
\end{array}\right]+\left[\begin{array}{c}
0 \\
p \omega_{r} \psi_{p m} \\
0
\end{array}\right] .
$$

Flux linkage equations are

$$
\left[\begin{array}{l}
\psi_{d} \\
\psi_{q}
\end{array}\right]=\left[\begin{array}{ccc}
L_{d} & 0 & M_{s f} \\
0 & L_{q} & 0
\end{array}\right]\left[\begin{array}{c}
i_{d} \\
i_{q} \\
i_{f}
\end{array}\right]+\left[\begin{array}{c}
\psi_{p m} \\
0
\end{array}\right] .
$$

Torque equations are

$$
T_{e}=\frac{3}{2} p\left(i_{q} \psi_{d}-i_{d} \psi_{q}\right)=\frac{3}{2} p i_{q}\left[\psi_{p m}+i_{d}\left(L_{d}-L_{q}\right)+M_{s f} i_{f}\right]
$$

where the meanings of the symbols are the following: $u_{d}, u_{q}$, $i_{d}$, and $i_{q}$ are the voltage and current components of the $d$ -

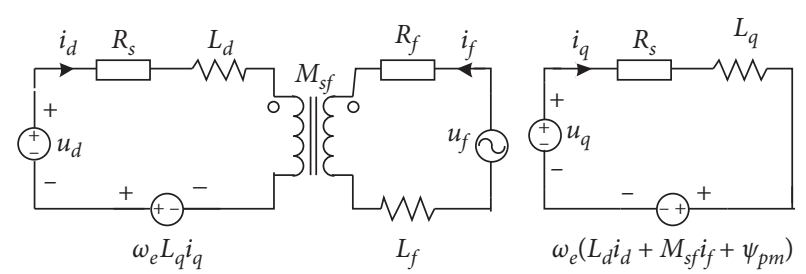

FIgURE 1: The equivalent circuit model of the $d$-q-axis of the HESM.

and $q$-axes, respectively; $\psi_{p m}, \psi_{d}$, and $\psi_{q}$ are the permanent magnet flux linkage and the flux linkages of the $d$ - and $q$ axes, respectively; $u_{f}$ and $i_{f}$ are the voltage and current of the excitation coils; $R_{s}$ and $R_{f}$ are the resistances of armature and excitation winding, respectively; $L_{d}, L_{q}$, and $M_{s f}$ are the armature $d$ - and $q$-axes inductance and the mutual inductance between the armature and the excitation winding, respectively; and $\omega_{r}$ is the angular velocity, $p$ is the pole pairs of the motor, and $T_{e}$ is the electromagnetic torque.

\section{Speed Partition Optimization Control of the HESM}

3.1. Introduction of the Control Strategy. In analogy with the PMSM motor, the speed range may be divided into two regions, as shown in Figure 2, in which the partition control method is adopted. Region I $\left(\omega_{r} \leq \omega_{N}\right.$, where $\omega_{r}$ is the motor speed and $\omega_{N}$ is the rated speed of the motor) is the lowspeed region, where the control method of flux-strengthening or no-excitation is applied. The motor in this speed range can obtain the excess electromagnetic torque without exceeding the current limit. Moreover, when the motor speed is less than or equal to the flux-strengthening basespeed $\omega_{M}$, the maximum output electromagnetic torque reaches $T_{\max }$, above which the output torque will decrease linearly due to the voltage limitation on the motor. Region II $\left(\omega_{r}>\omega_{N}\right)$ is the high-speed region, in which the back EMF of the motor is kept constant. By suitably adjusting the excitation current and the $d$-axis current, we can implement the flux-weakening speed regulation, making the motor able to operate at speed much higher than the rated speed.

3.2. Control Method in the Low-Speed Region $\left(\omega_{r} \leq \omega_{N}\right)$. According to the HESM electromagnetic properties, if there is no-excitation current, the reference torque current $i_{\text {Tref }}$ at the output of the speed controller is exactly the torque component of the armature current. In other words, in the low-speed region, an HESM is equivalent to a PMSM with a similar structure and the vector control method with $i_{d}=0$ may be used. Upon exploiting the torque equilibrium relation, we have that when the HESM is under the control of flux-strengthening, the reference value of electromagnetic torque is given by

$$
T_{e}^{*}=\frac{3}{2} p\left(\psi_{p m}+M_{s f} i_{f \mathrm{ref}}\right) i_{q \mathrm{ref}}=\frac{3}{2} p \psi_{p m} i_{T \mathrm{ref}},
$$

where $i_{\text {fref }}$ is the reference value of the excitation current and $i_{\text {qref }}$ is the reference value of the $q$-axis current. The basic current distribution may be obtained from (4) as follows: 


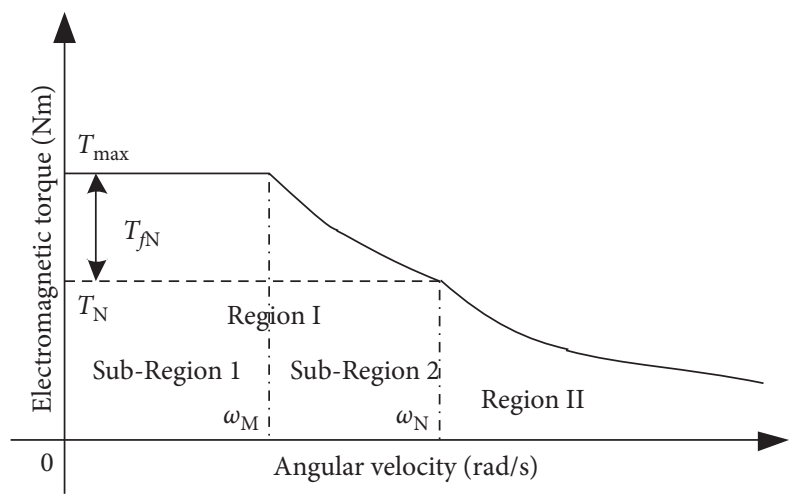

FIGURE 2: Schematic diagram of the speed partition control of the HESM.

$$
i_{\text {qref }}=\frac{\psi_{p m} i_{\text {Tref }}}{\psi_{p m}+M_{s f} i_{f \text { ref }}} .
$$

By inspecting (4), we see that when the flux-strengthening excitation current and the $q$-axis current take the values $i_{f \text { ref }}=0$ and $i_{q \text { ref }}=I_{q N}$ or $i_{\text {fref }}=I_{f N}$ and $i_{q \text { ref }}=I_{q N}$ (where $I_{f N}, I_{q N}$ are the excitation current and the rated $q$-axis current), the reference electromagnetic torques at the speed controller are the rated value and the maximum value, respectively:

$$
\left\{\begin{array}{l}
T_{N}=\frac{3}{2} p \psi_{p m} I_{q N} \\
T_{\max }=\frac{3}{2} p\left(\psi_{p m}+M_{s f} I_{f N}\right) I_{q N} .
\end{array}\right.
$$

In (5), since $i_{\text {qref }}$ and $i_{\text {fref }}$ are both unknown, one more constraint equation should be added to obtain the unique value of the current distribution of the HESM in the lowspeed region. In this study, we take the minimization of the copper loss by the motor as the other constraint condition to solve the two reference values of the current. Upon considering the $i_{d}=0$ vector control method, the equation of the copper loss for the HESM is given by

$$
P_{c u}=\frac{3}{2} R_{s} i_{q}^{2}+R_{f} i_{f}^{2}
$$

The Lagrangian multiplier method is then adopted to determine the minimum value of $P_{c u}$. By combining (4) and (7) and letting $i_{q}=i_{\text {qref }}, i_{f}=i_{\text {fref }}$, we obtain the Lagrangian function as follows:

$$
L\left(i_{q}, i_{f}, \lambda\right)=P_{c u}+\lambda\left[\frac{3}{2} p\left(\psi_{p m}+M_{s f} i_{f \text { ref }}\right) \cdot i_{q r e f}-\frac{3}{2} p \psi_{p m} i_{T \text { ref }}\right] .
$$

Taking the derivatives of (8) with respect to $i_{\text {qref }}$ and $i_{\text {fref }}$ and setting them to zero, we can eliminate $\lambda$ and get the relation between $i_{\text {qref }}$ and $i_{\text {fref }}$ when the minimum copper loss condition is satisfied:

$$
2 R_{f} i_{f \text { ref }}\left(\psi_{p m}+M_{s f} i_{f \text { ref }}\right)-3 R_{s} M_{s f} i_{q \text { ref }}^{2}=0 .
$$

By substituting (5) into (9), we obtain the following equation after straightforward algebra:

$$
\begin{aligned}
& 2 R_{f} M_{s f}^{3} i_{f \text { ref }}^{4}+6 R_{f} M_{s f}^{2} \psi_{p m} i_{f \text { ref }}^{3}+6 R_{f} M_{s f} \psi_{p m}^{2} i_{f \text { ref }}^{2} \\
& \quad+2 R_{f} \psi_{p m}^{3} i_{f \text { ref }}-3 R_{s} M_{s f} \psi_{p m}^{2} i_{T \text { ref }}^{2}=0 .
\end{aligned}
$$

Equation (10) is a quartic equation with one unknown variable which can be solved numerically, e.g., by Newtonian iteration. When the HESM is operating in the low-speed region, the motor has only two states: the flux-strengthening and no-excitation states. We thus have $i_{\text {fref }} \geq 0$, and we may assume $i_{\text {fref }}=0.5 I_{f N}$ without loss of generality. Simulations and experiments both verify that four iterations are enough to obtain the approximate solution of $i_{\text {fref }}$ with a relative error lower than $1 \%$. Therefore, the real-time operation requirement can be satisfied when using the DSP to control the HESM. Finally, by substituting the value of $i_{\text {fref }}$ into (5), we find $i_{q \text { ref. }}$

In the above calculations, since the speed is low, the fluxstrengthening is not leading to a very large back EMF and, in turn, there is no need to consider the limitation of bus voltage. On the other hand, when the speed exceeds $\omega_{M}$ and the system enters into sub-Region 2 (see Figure 2), the torque current reference value should be limited in order to prevent overloading. By combining (5) and (6), we obtain $\omega_{M}$, as well as the value of the maximum torque current reference $I_{T \max }$ :

$$
\left\{\begin{array}{l}
\omega_{M}=\frac{P_{N}}{T_{\max }}=\frac{2 P_{N}}{3 p I_{q N}\left(\psi_{p m}+M_{s f} I_{f N}\right)}, \\
I_{T \max }= \begin{cases}I_{q N}\left(1+I_{f N} \frac{M_{s f}}{\psi_{p m}}\right), & \omega_{r} \leq \omega_{M}, \\
I_{q N}\left(1+I_{f N} \frac{M_{s f}}{\psi_{p m}}\right) \frac{\omega_{M}}{\omega_{r}}, & \omega_{M}<\omega_{r} \leq \omega_{N},\end{cases}
\end{array}\right.
$$

where $P_{N}$ is the rated power of the HESM.

\subsection{Control Method in the High-Speed Region}

3.3.1. Basic Algorithm. In the design of the HESM control system, the flux-weakening control in the high-speed region is a challenging crucial step. When the motor speed reaches the rated speed $\omega_{N}$, its back EMF approaches the voltage value at the armature end, and to further increase the speed, the flux-weakening control should be applied. In this condition, the regulation of the HESM armature current and excitation current are constrained by the voltage limit cycle, as it happens with the flux-weakening speed regulation mode in the constant power region of the PMSM. Under the steady operating condition, the voltage vector amplitude should satisfy

$$
u_{s}^{2}=u_{d}^{2}+u_{q}^{2} \leq U_{\mathrm{lim}}^{2}
$$


where $U_{\text {lim }}$ is the limiting value of the voltage vector due to the back EMF at the motor side. Neglecting the resistance of the armature coil, the $d-q$ back EMF components for a steady-state motor are given by

$$
\left\{\begin{array}{l}
u_{d}=-p \omega_{r} L_{q} i_{q}, \\
u_{q}=p \omega_{r}\left(L_{d} i_{d}+M_{s f} i_{f}+\psi_{p m}\right) .
\end{array}\right.
$$

Substituting (13) into (12), we obtain

$$
\left(p \omega_{r} L_{q} i_{q}\right)^{2}+p^{2} \omega_{r}^{2}\left(L_{d} i_{d}+M_{s f} i_{f}+\psi_{p m}\right)^{2} \leq U_{\mathrm{lim}}^{2} .
$$

Upon inspecting the above formula, it is straightforward to see that applying the flux-weakening control on the HESM, the current distribution is limited by $U_{\text {lim }}$, while the $q$-axis component of the back EMF is given by

$$
E_{q}=p \omega_{r}\left(L_{d} i_{d}+M_{s f} i_{f}+\psi_{p m}\right) .
$$

By combining (14) and (15), we have that when the HESM is operating at no-load speed, $i_{q} \approx 0$ and (14) can be simplified to

$$
E_{q} \leq U_{\lim }
$$

Overall, the principle of the flux-weakening speed regulation of HESM is very much similar to the PMSM one, and we only need to keep $E_{q}$ smaller than or equal to $U_{\lim }$ when applying the flux-weakening control.

It can be seen from (15) that when $i_{d}=i_{f}=0, \omega_{r}$ depends linearly on $E_{q}$. As a consequence, when the normal vector control is applied, the maximum no-load speed $\omega_{\max }$ at the no-excitation current is linearly dependent on the DC bus voltage $U_{d c}$. The flux-weakening base-speed $\omega_{B d e c}$ is limited by $\omega_{\max }$, whose value can be obtained by the $i_{d}=i_{f}=0$ vector control under the no-load experimental condition. After collecting experimental data of bus voltage and maximum no-load speed, we build a linear fitting

$$
\left\{\begin{array}{l}
\omega_{\max }=k_{v} U_{d c}+\omega_{0}, \\
\omega_{B d e c}=k_{b} \omega_{\max },
\end{array}\right.
$$

where $k_{v}$ is the ratio of the maximum speed over the voltage; $\omega_{0}$ is the speed offset value; and $k_{b}$ is the flux-weakening base-speed coefficient. In order to simplify calculation, we may set the flux-weakening base speed to $\omega_{B d e c}=\omega_{N}$, so $k_{b}=\omega_{N} / \omega_{\max }$.

In the process of flux-weakening speed regulation, a control strategy to keep the back EMF invariant should be adopted. This is in order to ensure that the back EMF of the motor is smaller than the voltage of the power source and, in turn, to prevent the saturation of the current controller and the loss of control on the speed. To this aim, a strategy similar to the flux-weakening control in the PMSM may be employed. The back EMF base value $E_{\text {base }}$ is approximately

$$
E_{\text {base }}=p \omega_{\text {Bdec }} \psi_{p m}<U_{\text {lim }} \text {. }
$$

From (15), the motor back EMF may be approximated by

$$
E_{\mathrm{back}} \approx E_{q}=p \omega_{r}\left(\psi_{p m}+L_{d} i_{d}+M_{s f} i_{f}\right) .
$$

To maintain the back EMF nearly constant in the highspeed region and under the flux-weakening speed regulation, the estimated value of the back EMF should satisfy

$$
E_{\text {back }}=E_{\text {base }} \text {. }
$$

Combining (18)-(20) and setting the $d$-axis current and excitation current at their reference values, respectively, i.e., $i_{d}=i_{d \text { ref }}, i_{f}=i_{\text {fref }}$, we can obtain the voltage constraint equation of the HESM flux-weakening speed regulation. After some algebra, we arrive at

$$
L_{d} i_{d \mathrm{ref}}+M_{s f} i_{f \mathrm{ref}}=\psi_{p m}\left(\frac{\omega_{B d e c}}{\omega_{r}}-1\right) .
$$

Obviously, (21) is different from the analogue voltage limit equations of the normal PMSM because it is an approximate equation. Its precision and the practical control effect are determined by the value of $\omega_{B d e c}$. We will discuss this point later.

In the operating process of flux-weakening, to control and minimize the copper loss of the HESM, the limitation of the loading torque and the inverter output voltage should be satisfied simultaneously. That is, under the condition posed by (3) and (21), we need to find the value of the current reference needed to minimize the copper loss of the motor by solving the corresponding loss equation:

$$
P_{c u}=\frac{3}{2} R_{s}\left(i_{d}^{2}+i_{q}^{2}\right)+R_{f} i_{f}^{2}
$$

Combining equations (3), (21), and (22) and setting $P_{c u}$, $i_{d}, i_{q}$, and $i_{f}$ to their reference values, i.e., $P_{c u}=P_{c u \_ \text {ref }}$, $i_{d}=i_{d \mathrm{ref}}, i_{q}=i_{q \mathrm{ref}}$, and $i_{f}=i_{\text {fref }}$, we can construct the Lagrangian function as follows:

$$
\begin{aligned}
& L\left(i_{d \mathrm{ref}}, i_{q \mathrm{ref}}, i_{f \mathrm{ref}}, \lambda_{1}, \lambda_{2}\right)=P_{c u_{\text {ref }}}+\lambda_{1}\left\{\frac{3}{2} p i_{\text {qref }}\left[\psi_{p m}+i_{d \mathrm{ref}}\left(L_{d}-L_{q}\right)+M_{s f} i_{f \mathrm{ref}}\right]-T_{\text {eref }}\right\}+\lambda_{2}\left[L_{d} i_{d \mathrm{ref}}+M_{s f} i_{f \mathrm{ref}}-\psi_{p m}\left(\frac{\omega_{B d e c}}{\omega_{r}}-1\right)\right] \\
& =\frac{3}{2} R_{s}\left(i_{d \mathrm{ref}}^{2}+i_{q \mathrm{ref}}^{2}\right)+R_{f} i_{f \mathrm{ref}}^{2}+\lambda_{1}\left\{\frac{3}{2} p i_{\text {qref }}\left[\psi_{p m}+i_{d \mathrm{ref}}\left(L_{d}-L_{q}\right)+M_{s f} i_{f \mathrm{ref}}\right]-T_{\text {eref }}\right\} \\
& +\lambda_{2}\left[L_{d} i_{d \mathrm{ref}}+M_{s f} i_{f \mathrm{ref}}-\psi_{p m}\left(\frac{\omega_{B d e c}}{\omega_{r}}-1\right)\right],
\end{aligned}
$$


where $\lambda_{1}$ and $\lambda_{2}$ are the Lagrangian multipliers. Upon eliminating the multipliers, we arrive at the following equation:

$$
\begin{aligned}
& -3 p R_{s}\left(L_{d}-L_{q}\right) i_{d \mathrm{ref}}^{2}-3 p R_{s} L_{d} i_{q \mathrm{qref}}^{2}+2 p L_{d} R_{f} i_{f \mathrm{ref}}^{2} \\
& +3 p R_{s}\left(L_{d}-L_{q}\right) i_{d \mathrm{ref}} i_{q \mathrm{ref}}+\frac{p}{M_{s f}}\left[2 R_{f} L_{d}\left(L_{d}-L_{q}\right)\right. \\
& \left.-3 R_{s} M_{s f}^{2}\right] i_{d \mathrm{ref}} i_{f \mathrm{ref}}-3 p R_{s} \psi_{p m} i_{d \mathrm{ref}}+\frac{2 p L_{d} R_{f} \psi_{p m}}{M_{s f}} i_{f \mathrm{ref}}=0 .
\end{aligned}
$$

In addition, by combining (3) and (21) and using an iteration method [13], we may find all the current reference values $i_{\text {dref }}, i_{\text {qref }}$, and $i_{\text {fref }}$ leading to the minimization of the copper loss by the motor.

Equations (3), (21), and (24) form a system of equations with three unknown variables. Although it can be solved via the iteration method, the calculation process may be involved, and in practical engineering applications, a simplified control algorithm for the minimization of copper loss may be better adopted.

3.3.2. Simplified Control Algorithm. According to (2) and (19), the larger is the flux-weakening current, the weaker is the air gap magnetic field and the smaller is the back EMF. Overall, this results in a larger $q$-axis current required at a given load, and, therefore, to realize the copper loss minimization control, the flux-weakening current should be reduced as much as possible to make the back EMF as close as possible to the terminal voltage. The $q$-axis current reference value is given by the speed controller and can be adjusted automatically according to the variation of the load and speed:

$$
i_{\text {qref }}=k_{i} T_{\text {eref }},
$$

where $T_{\text {eref }}$ is the reference electromagnetic torque output by the speed controller and $k_{i}$ is the ratio coefficient of the current over the torque, i.e., a constant determined by the HESM electromagnetic properties.

Since $i_{d}, i_{q}$, and $i_{f}$ are fully decoupled, they do not need a unified calculation. Therefore, when applying the control algorithm for the copper loss minimization, we can use the incomplete copper loss equation in place of (22) to conduct the calculation; that is,

$$
P_{c u}=\frac{3}{2} i_{d}^{2} R_{s}+i_{f}^{2} R_{f},
$$

leading to a simplified algorithm of the copper loss minimization control. The differences between this algorithm and the original one are about the current calculation process. In the present algorithm, only the voltage constraint in (21) is used while the torque equation is not included. Needless to say, this considerably simplifies calculations. Combining equations (21) and (26) and setting $P_{c u}, i_{d}$, and $i_{f}$ to their reference values, we obtain the following Lagrangian function:

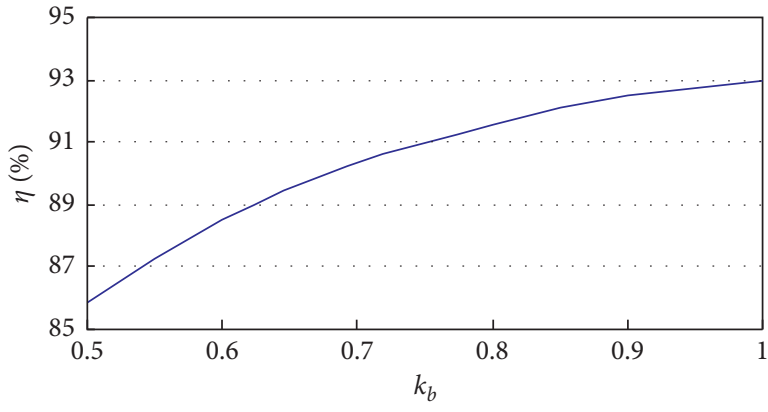

FIGURE 3: Relations between efficiency and the coefficient of fluxweakening base speed.

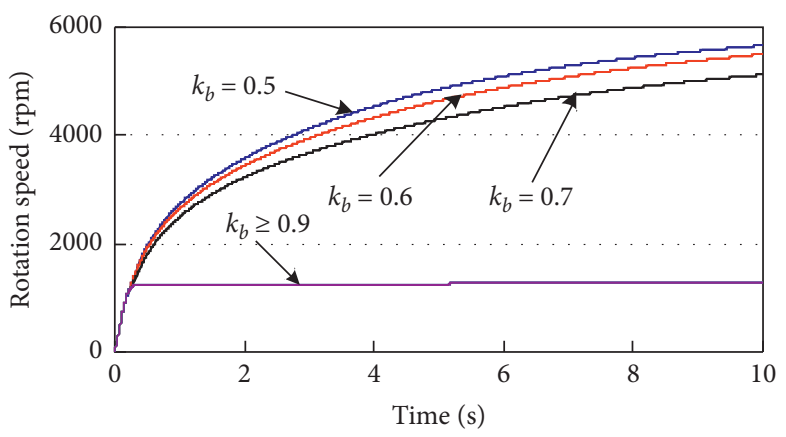

FIGURE 4: Speed rising curves as a function of time for different $k_{b}$.

$$
\begin{aligned}
L\left(i_{\text {dref }}, i_{\text {fref }}, \lambda\right)= & P_{\text {cu_ref }}+\lambda\left[L_{d} i_{\text {dref }}+M_{s f} i_{\text {fref }}-\psi_{p m}\left(\frac{\omega_{\text {Bdec }}}{\omega_{r}}-1\right)\right] \\
= & \frac{3}{2} i_{\text {dref }}^{2} R_{s}+i_{\text {fref }}^{2} R_{f} \\
& +\lambda\left[L_{d} i_{\text {dref }}+M_{s f} i_{\text {fref }}-\psi_{p m}\left(\frac{\omega_{\text {Bdec }}}{\omega_{r}}-1\right)\right] .
\end{aligned}
$$

Using (21) and taking the derivatives of the above equation with respect to $i_{d r e f}$ and $i_{\text {fref }}$, we get the following solution:

$$
\left\{\begin{array}{l}
i_{\text {dref }}=\frac{2 L_{d} R_{f} \psi_{p m}}{2 L_{d}^{2} R_{f}+3 R_{s} M_{s f}^{2}}\left(\frac{\omega_{\text {Bdec }}}{\omega_{r}}-1\right), \\
i_{\text {fref }}=\frac{3 R_{s} M_{s f}}{2 L_{d} R_{f}} i_{\text {ref }},
\end{array}\right.
$$

which, by using the notation

$$
\left\{\begin{array}{l}
k_{d}=\frac{2 L_{d} R_{f} \psi_{p m}}{2 L_{d}^{2} R_{f}+3 R_{s} M_{s f}^{2}}, \\
k_{f d}=\frac{3 R_{s} M_{s f}}{2 L_{d} R_{f}},
\end{array}\right.
$$

can be rewritten as 


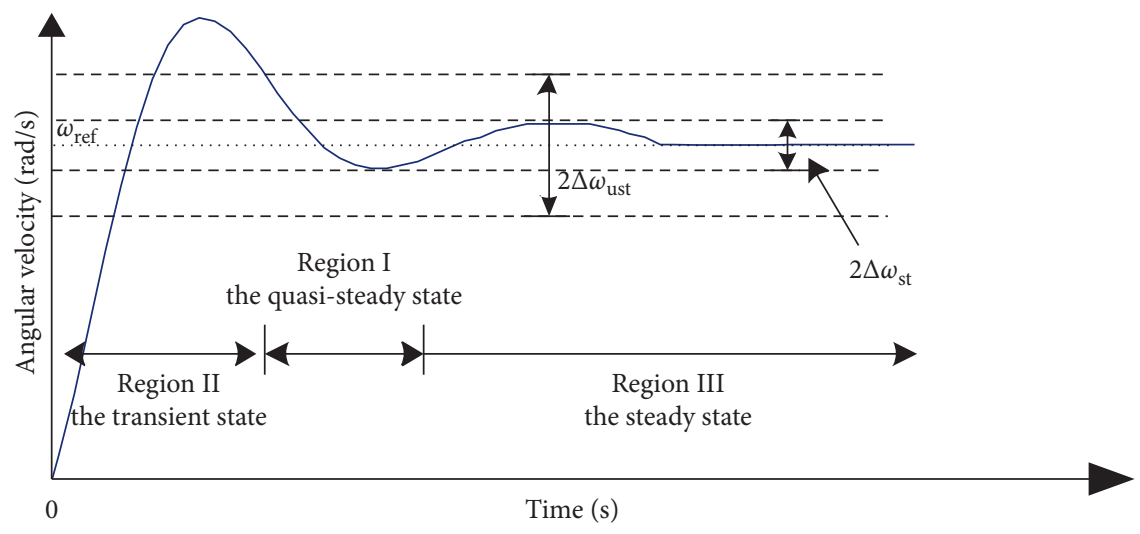

FIGURE 5: Partition of operational regimes of HESM.

$$
\left\{\begin{array}{l}
i_{d \text { ref }}=k_{d}\left(\frac{\omega_{B d e c}}{\omega_{r}}-1\right), \\
i_{f \text { ref }}=k_{f d} i_{d \text { ref }} .
\end{array}\right.
$$

From (30), we see that the flux-weakening currents $i_{d r e f}$ and $i_{\text {fref }}$ decrease when $\omega_{B d e c}$ increases, which in turn leads to a decrease of the motor flux weakening. Therefore, by increasing or decreasing the value of $\omega_{B d e c}$, one may tune the motor flux-weakening speed and the copper loss magnitude.

\subsubsection{Self-Adaptive Regulation of Flux-Weakening Base-} Speed Coefficient. From the above theoretical analysis, we know that $\omega_{B d e c}$ influences the operating properties of the HESM flux-weakening control. Those effects may be summarized as follows: (1) when $\omega_{B d e c}$ is too large, the fluxweakening current is too small, thus leading to an insufficient flux-weakening. In this case, the HESM speed cannot reach the set value, i.e., we have a limitation on the speed range; (2) when $\omega_{B d e c}$ is too small, the flux-weakening current is too large, leading to overweakening of the flux in the motor and, in turn, to an increase of the copper loss, resulting in a low efficiency. Therefore, in order to ensure optimal control of the motor flux-weakening speed regulation, further analysis on the influence of $\omega_{\text {Bdec }}$ should be performed, possibly leading to a suitable choice of its value.

From (17), we know that the magnitude of $\omega_{B d e c}$ is determined by the flux-weakening base-speed coefficient $k_{b}$. Therefore, we may describe the relationship among the variables as follows:

$$
\begin{aligned}
k_{b} \uparrow & \Longrightarrow \omega_{\text {Bdec }} \uparrow \Longrightarrow E_{\text {base }} \uparrow,\left(\left|i_{\text {dref }}\right|,\left|i_{\text {qref }}\right|,\left|i_{\text {fref }}\right|\right) \downarrow \\
& \Longrightarrow\left(\left|i_{d}\right|,\left|i_{q}\right|,\left|i_{f}\right|\right) \downarrow \Longrightarrow P_{c u} \downarrow .
\end{aligned}
$$

In other words, the crucial ingredient to determine the optimal $\omega_{B d e c}$ value and to jointly realize the optimizations of the motor speed range and the efficiency is the choice of the value for $k_{b}$.

In order to assess the influence of $k_{b}$ on the HESM efficiency, we may use the system simulation analysis while changing its value. In the simulation, all the electrical parameters are set according to the prototype practical parameters [14]. The simulation result is presented in Figure 3, which indicates that the motor efficiency increases monotonically with $k_{b}$.

To gain a deeper insight into the influence of $k_{b}$ on the HESM speed range and dynamical properties, we have performed a set of simulated experiments by setting different $k_{b}$ values in the HESM control system. The behavior of the speed as a function of time is illustrated in Figure 4 for different values of $k_{b}$. Results have been obtained by setting the load at $0.5 \mathrm{Nm}$ and the reference speed at $6000 \mathrm{rpm}$, and they indicate that the larger is $k_{b}$, the lower is the maximum speed that the motor may reach. When $k_{b} \geq 0.9$, the maximum speed is smaller than $1500 \mathrm{rpm}$. The value $k_{b}$ also influences the dynamical properties of the motor flux-weakening speed regulation. In particular, the larger is $k_{b}$, the slower is the speed rising and the longer is the starting time.

In conclusion, we have that decreasing $k_{b}$ may enhance the HESM speed range and dynamical properties, however at the expense of reducing the efficiency. On the contrary, by increasing $k_{b}$, we reduce the copper loss of the motor and enhance its efficiency, but the speed range decreases, and the flux-weakening speed regulation dynamical properties degrades. In particular, when $k_{b}$ exceeds a certain threshold value, the flux-weakening base speed becomes too high and the motor becomes unable to switch to the flux-weakening speed regulation region, resulting in a large decrease of the speed range. In other words, we have that keeping $k_{b}$ constant when the motor is operating does not guarantee the optimality of its performance under different working conditions.

In order to solve these issues, this paper proposes an HESM control method which may self-adaptively adjust the flux-weakening base-speed coefficient $k_{b}$ according to the working condition, thus resulting in an enhancement of the motor overall performance. The principle is to split the HESM operating regimes in the high-speed region into three regions, which are the region of transient state, the region of quasisteady state, and the region of steady state, respectively. The three regions are determined by the difference between the set speed and the practical speed and are illustrated in Figure 5. According to the changes in the motor state, we can 


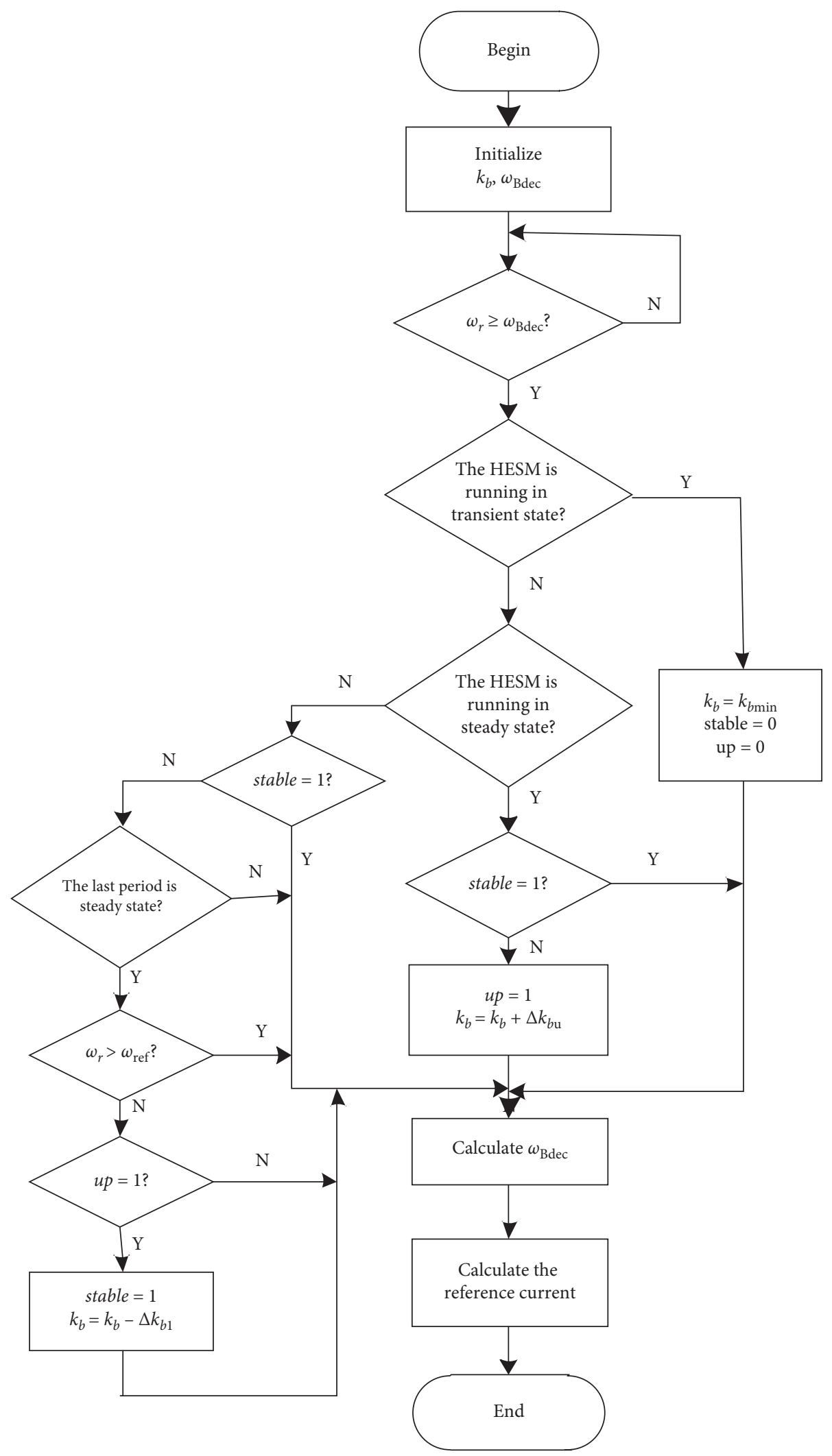

FIGURE 6: Flow chart of adaptive regulating $k_{b}$.

dynamically adjust the coefficient $k_{b}$ to optimize the motor overall performance, as shown in Figure 6. The regulation method for $k_{b}$ in each region is illustrated below.
The region of transient state corresponds to $\left|\omega_{r}-\omega_{\text {ref }}\right|>\Delta \omega_{u s t}$, where $\Delta \omega_{\text {ust }}$ is the error upper limit in the region of quasisteady state. The parameters $k_{b}$, stable, and $u p$ 


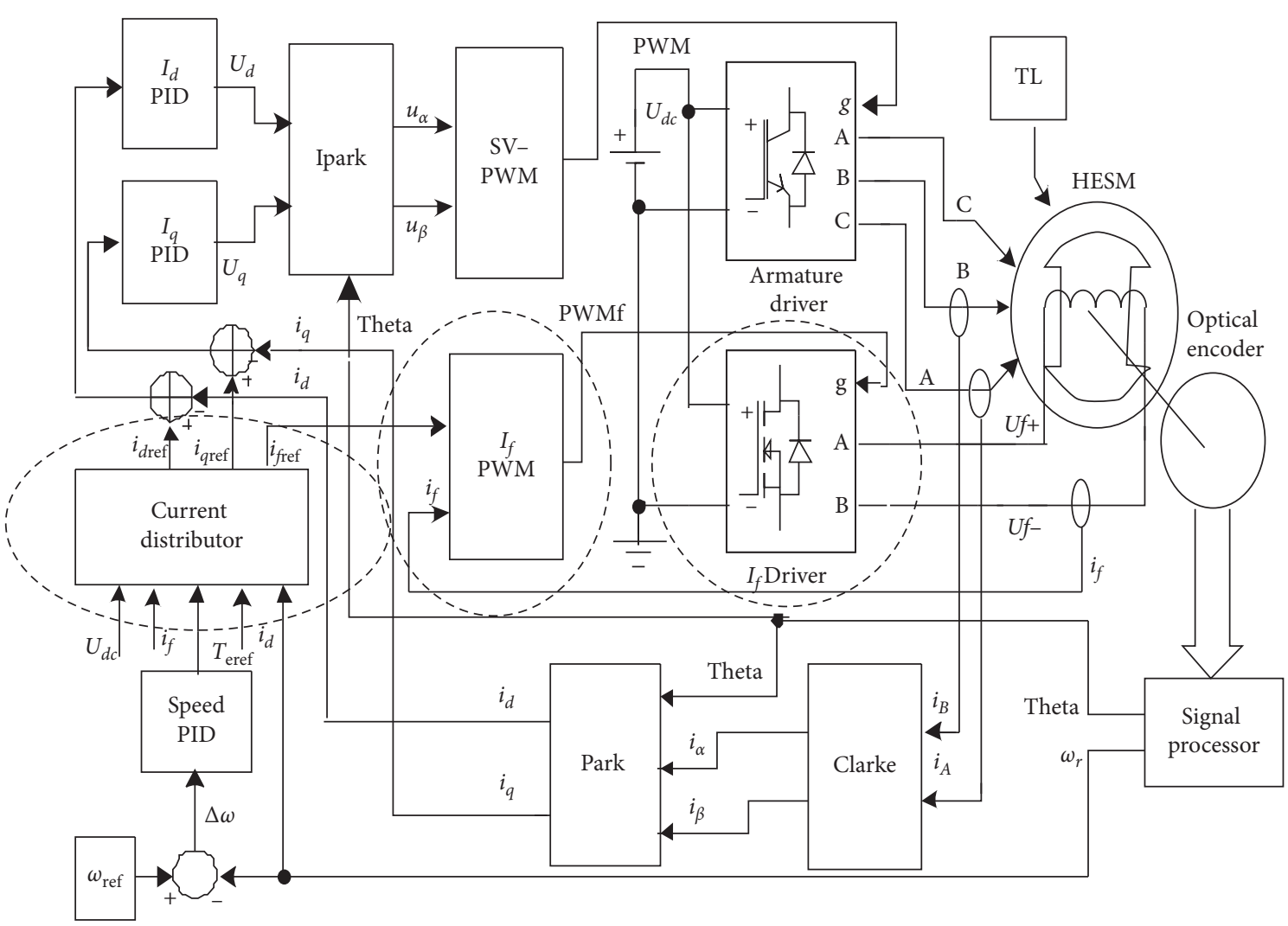

Figure 7: HESM control system model.

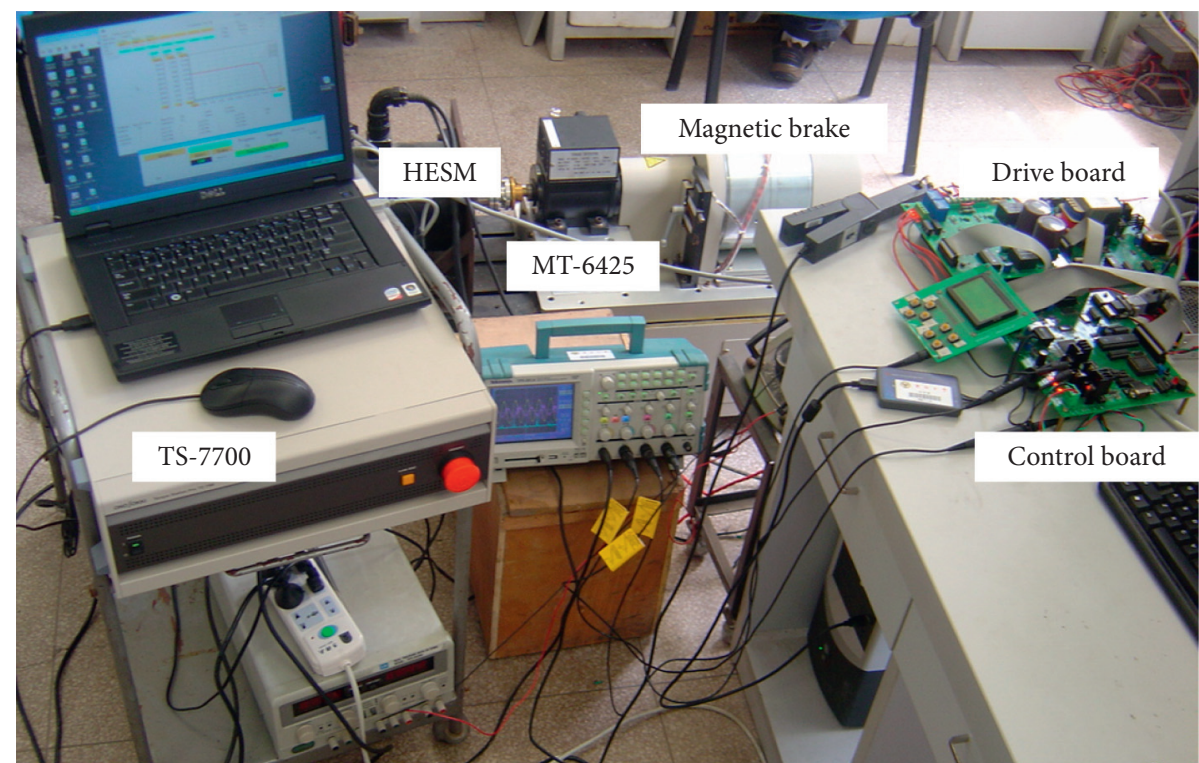

FIGURE 8: Experiment system of HESM.

are initialized and kept constant in this region. Since to improve the HESM speed range and dynamic property, the initial value $k_{b \min }$ should not be too large, we set it at $k_{b \min }=0.5$.

The region of quasisteady state corresponds to the range $\Delta \omega_{s t}<\left|\omega_{r}-\omega_{\text {ref }}\right| \leq \Delta \omega_{\text {ust }}$, where $\Delta \omega_{s t}$ is the error upper limit in the region of the steady state. In this region, according to the values of stable, up and the magnitude of $\omega_{r}$, one may decide whether to make an adjustment of $k_{b}$ or not.

The region of the steady state corresponds to $\left|\omega_{r}-\omega_{\text {ref }}\right| \leq \Delta \omega_{s t}$ : this region may be further divided in two subcases according to the value of stable, which indicates 
whether the optimization is complete or not. If it is equal to 1 , then the regulation of $k_{b}$ is completed and the value should be kept unchanged. If instead it is equal to 0 , then the optimization is not completed and $k_{b}$ should be increased, along with the flag set to $u p=1$, indicating that the adaptive control should be continued.

In the process of self-adaptive regulation of $k_{b}$, the HESM is kept in the steady operating state until $k_{b}$ exceeds the upper limit, then the motor is forced to lower its speed and leave the steady state. Therefore, the $k_{b}$ regulation step length, termed $\Delta k_{b u}$, cannot be too large to avoid the generation of large overshoot. At the same time, the regulation time period should be properly set according to the motor electrical and mechanical properties. This is in order to keep the armature current and excitation current variation amplitude small and smooth and also to keep the output electromagnetic torque nearly constant in the whole regulation process.

During the operating process of the HESM, due to the influence from the set speed, load variation, or other external factors, the motor is forced to leave the region of steady state, go across the region of quasisteady state, and finally reach the region of transient state, where $k_{b}$ should be reset to a small initial value $k_{b \min }$. On the other hand, when the motor reaches again the steady state, $k_{b}$ should be automatically adjusted to optimize the motor efficiency.

\section{Analysis of the Experimental Results}

4.1. Control System Modeling. The HESM vector control system model, resulting from the above theoretical analysis based on the speed partition and vector control principle, is shown in Figure 7. This control system model is similar to the regular PMSM vector control one, however with three more functional modules (contained in the dashed ellipses). The three modules are the current distributor, the excitation current pulse width modulation, and the excitation drive module. Among them, the current distributor is the core module of the HESM control system, and it is designed according to the speed partition control principle. Its main function is to estimate the armature current and the excitation current and then output the reference currents $i_{d r e f}$, $i_{\text {qref }}$ and $i_{\text {fref. }}$

4.2. Analysis of the Experimental Results. Figure 8 illustrates the results of the experiments performed with the HESM control system. The experiment adopts the expert system TS7700, from the Japanese Ono Measurement Device, for the torque workstation equipped with the torque sensor MT6425 , the eddy current brake, and so forth. This series of test devices can be used to measure the different parameters influencing the motor properties, including the speed, torque, input and output power, and efficiency. We employ the HESM prototype with a claw pole structure, which includes the TAMAGAWA OIH48-2500 increment photoelectric encoder. The parameters of HESM are given in Table 1. The control board is a hardware circuit based on the TMS320F2812 + AT89C55WD architecture, along with the two driver boards of armature and excitation, and a circuit
TABle 1: Parameters of HESM.

\begin{tabular}{lc}
\hline Parameters & Value \\
\hline$P_{N}(\mathrm{~W})$ & 700 \\
$n_{N}(\mathrm{rpm})$ & 500 \\
$T_{N}(\mathrm{Nm})$ & 13 \\
$M_{s f}(\mathrm{mH})$ & 76 \\
$\Psi_{p m}(\mathrm{~Wb})$ & 0.243 \\
$U_{d c}(\mathrm{~V})$ & 300 \\
$R_{s}(\Omega)$ & 2.7 \\
$R_{f}(\Omega)$ & 33.0 \\
$I_{f N}(\mathrm{~A})$ & 1.0 \\
$L_{f}(\mathrm{H})$ & 0.57 \\
$P$ & 4 \\
$L_{q}(\mathrm{mH})$ & 27 \\
$L_{d}(\mathrm{mH})$ & 38 \\
$I_{N}(\mathrm{~A})$ & 5 \\
\hline
\end{tabular}

board of buttons and display. The computer and the control board are connected to the DSP simulator by the JTAG interface. By applying the debugging software CCS3.3, we may also record the variation of the relevant electric parameters which are not accessible by other devices.

Figure 9 reports the experimental waveforms of HESM during the starting process. Figure 9(a) is the variation of speed and flux-weakening base-speed coefficient with time. Figure 9(b) shows the relationship between copper loss and time. In the experiment, the speed is set at $3000 \mathrm{rpm}$, and the load is set at $1 \mathrm{Nm}$. After the motor is switched on, as well as before the speed reaches the steady state, $k_{b}$ is kept nearly constant at the value $k_{b}=0.5$. After a transient time of about $2 \mathrm{~s}$, the motor enters the region of steady state and $k_{b}$ begins to linearly increase with time. In this regime, the speed is kept constant and the motor copper loss decreases as $k_{b}$ increases. After about $3.7 \mathrm{~s}, k_{b}$ reaches its peak. At this time, due to the overdecreasing of the excitation current and the $d$-axis current, the motor flux-weakening degree is insufficient, resulting in the increase of the motor back EMF and the saturation of the $q$-axis current controller, which will further force the speed to decrease and the motor to enter into the region of quasisteady state. After decreasing $k_{b}$ slightly, the motor returns to the steady state, and then the speed stays constant. The motor copper loss is instead slightly increasing during the callback of $k_{b}$. The results of this experiment indicate that the copper loss is about $60 \mathrm{~W}$ before the regulation of $k_{b}$ and about $40 \mathrm{~W}$ after the regulation of $k_{b}$, i.e., a reduction ratio of about $1 / 3$.

Figure 10 shows the experimental waveforms of the A-phase current and excitation current when the HESM is starting. In this experiment, the motor speed is set at $2500 \mathrm{rpm}$ and the load torque is given by $1.5 \mathrm{Nm}$. In order to enhance the motor performance when it starts up, we apply an excitation current to increase its starting torque $0.5 \mathrm{~s}$ before the motor is switched on. With the increase of the speed, $i_{f}$ quickly drops to 0 , and when the speed exceeds $n_{B d e c}, i_{f}$ becomes negative and the flux weakening is activated. When the motor speed enters the region of steady state, due to the gradual increasing of $k_{b}$, the excitation current and armature current decrease accordingly. Two seconds after the motor is started, a fluctuation with the duration of $0.5 \mathrm{~s}$ appears in the armature current and 


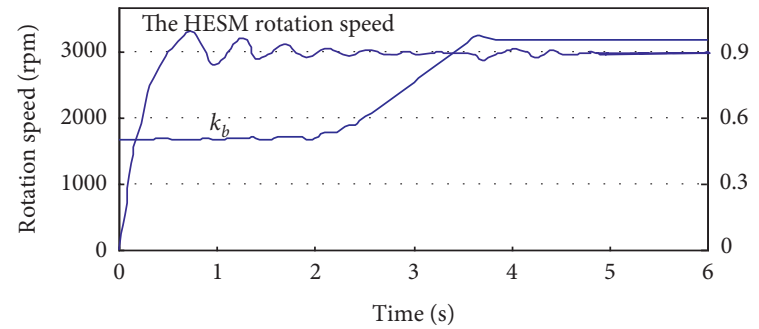

(a)

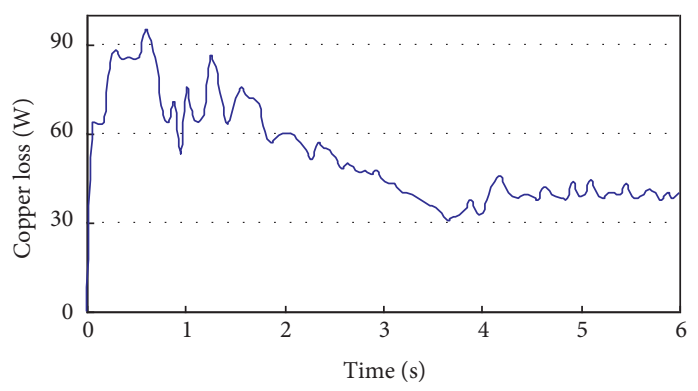

(b)

Figure 9: Experimental results of the copper loss minimization control for the HESM. (a) The relationship of rotate speed and $k_{b}$. (b) The relationship between copper consumption and time during motor startup.

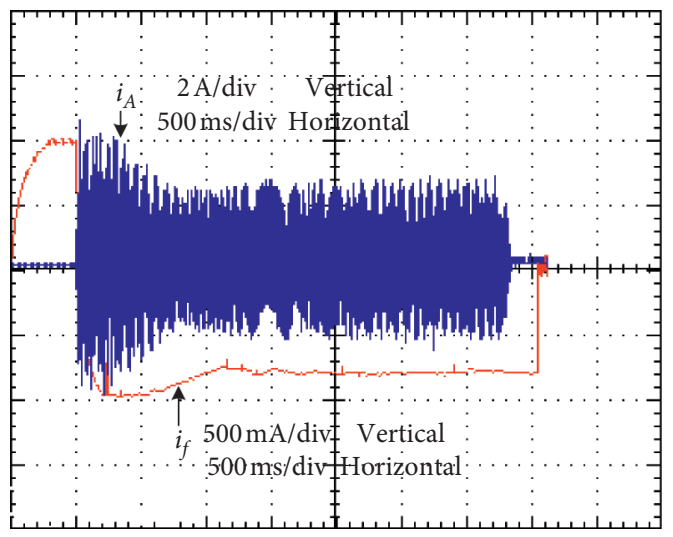

FIGURE 10: Starting current waveforms.

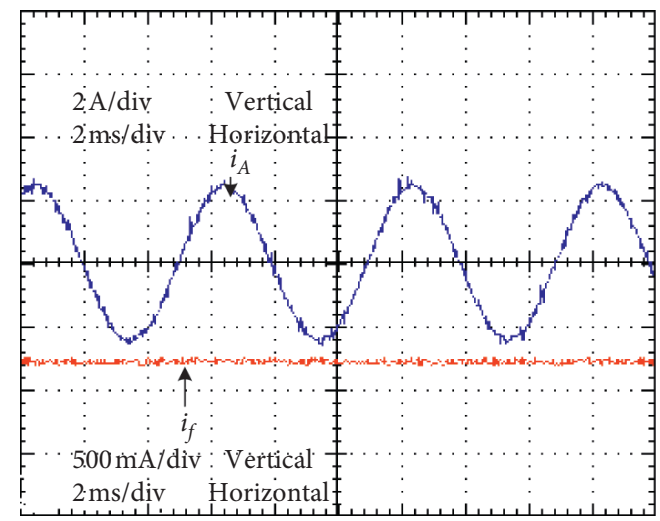

FIGURE 11: Steady-state current waveforms.

excitation current. This fluctuation is caused by overregulation of $k_{b}$, which leads to a decrease of the speed and drives the motor to the region of quasisteady state. At last, when we shut down the motor drive system because of the high operating speed, we need to slightly postpone the shutdown of the excitation current, in order to prevent an excessive response of the back EMF caused by the shutting down of the deep flux weakening. Therefore, as it may be seen in Figure 10, the armature current is shut down $0.3 \mathrm{~s}$ before the excitation current.
Figure 11 shows the current waveforms of the A-phase current and excitation current when HESM is operating in the steady state. The motor speed is here set at $2500 \mathrm{rpm}$ and the load torque is given by $1.5 \mathrm{Nm} . i_{f}$ is equal to $0.8 \mathrm{~A}$; it shows very small fluctuations, and it is relatively smooth. The phase current amplitude is $2.4 \mathrm{~A}$; it shows an almost sinusoidal waveform, and it has negligible harmonic components.

\section{Conclusion}

HESM represents a class of high-order, nonlinear, and strong-coupling systems with multiple variables. In this paper, upon building on the vector control method, we put forward a control algorithm which combines the copper loss minimization control algorithm with a self-adaptive method to dynamically regulate the flux-weakening base-speed coefficient and to further improve the motor's overall operation properties. We have assessed and verified the validity and efficiency of the proposed HESM control strategy by means of numerical simulations and experiments. The conclusions may be summarized as follows:

(1) For the HESM with salient pole (also effective for the hidden pole), we have proposed a basic copper loss minimization control algorithm. The calculations are relatively complex, but since we suitably exploit the regulation functions of armature current and excitation current on the torque and magnetic field, the motor efficiency optimization control may be still realized.

(2) Starting from the HESM electrical properties, we have proposed a simplified copper loss minimization control algorithm, which may largely simplify the calculation of the reference current. The method is similar to the basic copper loss minimization control algorithm.

(3) We have suggested a self-adaptive regulation of the flux-weakening base-speed coefficient for the motor operating in the steady state to optimize the control method of the flux-weakening current and, at the same time, to minimize the copper loss and enlarge the speed range. In this way, we have solved the problems arising when the HESM speed 
range, efficiency, and dynamical property cannot be simultaneously taken care of due to the constant flux-weakening base-speed coefficient. The overall performance and dynamical properties of the motor are significantly enhanced.

\section{Data Availability}

The data used to support the findings of this study are available from the corresponding author upon reasonable request.

\section{Conflicts of Interest}

The authors declare that they have no conflicts of interest regarding the publication of this paper.

\section{Acknowledgments}

This work was supported by the Natural Science Foundation of Henan Province (182300410224), the National Natural Science Foundation of China-Henan Joint Fund (U1804162), the Fundamental Scientific and Technological Research Funds of Henan Science and Technology Agency (192102210072 and 202102210109), and the Key Scientific Research Projects of Colleges and Universities in Henan Province (17A470008).

\section{References}

[1] C. C. Chan, K. T. Chau, J. Z. Jiang, W. Xia, M. Zhu, and R. Zhang, "Novel permanent magnet motor drives for electric vehicles," IEEE Transactions on Industrial Electronics, vol. 43, no. 2, pp. 331-339, 1996.

[2] Y. C. Wang, W. N. Fu, and S. X. Niu, "A novel structure of dual-stator hybrid excitation synchronous motor," IEEE Transactions On Applied Superconductivity, vol. 26, no. 4, pp. 421-425, 2016.

[3] H. May, R. Palka, P. Paplicki, S. Szkolny, and W.-R. Canders, "Modified concept of permanent magnet excited synchronous machines with improved high-speed features," Archives of Electrical Engineering, vol. 60, no. 4, pp. 531-540, 2011.

[4] H. J. Kim, D. Y. Kim, J. S. Jeong, and J.-P. Hong, "Proposition of structures for brushless hybrid-excitation synchronous motors with improved rotor," IEEE Transactions on Magnetics, vol. 52, no. 9, Article ID 8205415, 2016.

[5] Y. Amara, L. Vido, M. Gabsi, E. Hoang, A. Hamid Ben Ahmed, and M. Lecrivain, "Hybrid excitation synchronous machines: energy-efficient solution for vehicles propulsion," IEEE Transactions on Vehicular Technology, vol. 58, no. 5, pp. 2137-2149, 2009.

[6] S. Shinnaka, "New dynamic mathematical model and new dynamic vector simulators of hybrid-field synchronous motors," in Proceedings of the IEEE International Conference on Electric Machines and Drives, pp. 882-889, San Antonio, TX, USA, May 2005.

[7] S. M. Ahmad, P. T. João, and R. D. Maxime, "Hybridisation ratio for hybrid excitation synchronous motors in electric vehicles with enhanced performance," IET Electrical Systems in Transportation, vol. 8, no. 1, pp. 12-19, 2018.

[8] S. Shinnaka and T. Sagawa, "New optimal current control methods for energy-efficient and wide speed-range operation of hybrid-field synchronous motor," IEEE Transactions on Industrial Electronics, vol. 54, no. 5, pp. 2443-2450, 2007.

[9] W. J. Wang and Z. R. Zhang, "Maximum torque control of hybrid excitation synchronous machine drives based on field current self-optimizing method," in Proceedings of the 39th Annual Conference of the IEEE Industrial Electronics Society, pp. 2977-2982, Vienna, Austria, November 2013.

[10] F. G. Capponi, G. Borocci, D. Donato et al., "Flux regulation strategies for hybrid excitation synchronous machines," in Proceedings of the Energy Conversion Congress and Exposition (ECCE), 2014, pp. 4858-4865, IEEE, Pittsburgh, PA, USA, November 2014.

[11] S. M. Li and J. N. Liang, "Efficiency optimization control based on hybrid excitation synchronous motor," in Proceedings of the 22nd International Conference on Electrical Machines and Systems (ICEMS), pp. 310-314, Harbin, China, August 2019.

[12] M. M. Hang, X. J. Guo, P. Jin, H. Quanzhen, L. Yuping, and L. Na, "Flux-weakening control methods for hybrid excitation synchronous motor," Archives of Electrical Engineering, vol. 64, no. 3, pp. 427-439, 2015.

[13] Y. Zhang, Q. Z. Huang, M. M. Huang, D. Decker, and Y. Qing, "Design and experimental verfication of adaptive speed region control for hybrid excitation claw-pole synchronous machine," Progress In Electromagnetics Research C, vol. 88, pp. 195-205, 2018.

[14] M. Huang, H. Lin, H. Yunkai, P. Jin, and Y. Guo, "Fuzzy control for flux weakening of hybrid exciting synchronous motor based on particle swarm optimization algorithm," IEEE Transactions on Magnetics, vol. 48, no. 11, pp. 2989-2992, 2012.

[15] M. Huang, Y. Zhang, Q. Huang, X. Guo, and Y. Qing, "Hybrid excitation synchronous machine adaptive speed region control and experimental verification," International Journal of Applied Electromagnetics and Mechanics, vol. 58, no. 3, pp. 275-287, 2018. 